\title{
In-situ preparation of ultra-small Pt nanoparticles within rod-shaped mesoporous silica particles: 3-D tomography and catalytic oxidation of n-hexane
}

Laura Uson $^{[\mathrm{a}],[\mathrm{b}]}$, Jose L. Hueso ${ }^{[\mathrm{a}],[\mathrm{b}], *}$, Victor Sebastian ${ }^{[\mathrm{a}],[\mathrm{b}],{ }^{*}}$, Raul Arenal ${ }^{[\mathrm{c}],[\mathrm{d}]}$, Ileana Florea $^{[\mathrm{e}],[\mathrm{f}]}$, Silvia Irusta ${ }^{[\mathrm{a}],[\mathrm{b}]}$, Manuel Arruebo ${ }^{[\mathrm{a}],[\mathrm{b}]}$, and Jesus Santamaria ${ }^{[\mathrm{a}],[\mathrm{b}]}$

${ }^{a}$ Institute of Nanoscience of Aragon and Department of Chemical Engineering and Environmental Technology C/ Mariano Esquillor, s/n, 50018, Zaragoza (Spain).

*E-mail corresponding authors: jlhueso@unizar.es_victorse@unizar.es

${ }^{b}$ Networking Research Centre of Bioengineering, Biomaterials and Nanomedicine (CIBER-BBN), C/ Monforte de Lemos, 3-5 Pabellón 11, 28029 Madrid, (Spain).

${ }^{c}$ ARAID Foundation, Zaragoza (Spain)

${ }^{d}$ Laboratorio de Microscopias Avanzadas (LMA) and Instituto de Nanociencia de Aragón (INA), Edificio I+D, C/Mariano Esquillor, s/n, 50018 Zaragoza, (Spain)

${ }^{e}$ Laboratoire de Physique des Interfaces et des Couches Minces - LPICM École Polytechnique/CNRS, Route de Saclay,91128 Palaiseau Cedex, (France).

fIPCMS, UMR 7504 CNRS Université de Strasbourg, 23 rue du Loss BP 43, 67087 Strasbourg cedex 2, (France).

\begin{abstract}
The shape and porous configuration of supports are key parameters to design outstanding catalysts. However, the selection of a proper mesoporous support, such as SBA-15, by itself does not guarantee accessibility to catalytic sites. The distribution of the active phase and its stability are strongly related to the procedure used to deposit it
\end{abstract}


on the catalytic substrate. Herein, we have prepared rod-shaped SBA-15 silica supports functionalized with amine groups to facilitate the electrostatic attraction and a good distribution of the resulting Pt-based catalytic nanoparticles along the pore walls. Additionally, the use of tetrakis (hydroxymethyl) phosphonium chloride (THPC) as both reductant and stabilizer is presented as a novel alternative for $\mathrm{Pt}$ nanoparticle synthesis. The behaviour of this catalyst in the total oxidation of n-hexane demonstrates high activity and excellent stability after $70 \mathrm{~h}$ on reaction stream. STEM-HAADF and 3-D tomography were used to confirm the presence of the metallic nanoparticles within the mesochannels and to corroborate their reduced sintering after reaction.

Keywords: Platinum nanoparticles - SBA-15 rods - THPC • 3-D Tomography • oxidation $\bullet$ VOCs

\section{Introduction}

Volatile Organic Compounds (VOCs) are well-known contributors to air pollution. These VOCs may cause short and long-term adverse health effects, acting as greenhouse gases and contributing to photochemical smog [1]. The total oxidation of VOCs at mild temperatures is commonly carried out in the presence of active metalbased catalysts containing monometallic Pt or Pd or forming other alloys [2, 3], transition metal oxides $[4,5]$ or a combination of both types of elements $[6,7]$. Pt-based catalysts are generally preferred because of their excellent stability, high activity, good selectivity towards $\mathrm{CO}_{2}$ and higher resistance to poisoning and deactivation in comparison with metal oxide counterparts $[2,8]$. In terms of catalytic supports used for VOCs removal, Pt-exchanged zeolites and ordered mesoporous silica structures have been regarded as model alternatives to other commercial and industrial supports, such as 
alumina or silica $[2,9,10]$. SBA-15 supports have been particularly exploited in recent years in the oxidation of different VOCs such as toluene or the lessreactive n-hexane [8, 11]. These ordered mesoporous silica (OMS)-based materials display an ordered hexagonal array of pores and exhibit a higher hydrothermal and mechanical stability than other well-studied mesoporous ordered systems, such as MCM-41 [12].

A variety of synthesis procedures, such as incipient wetness impregnation, vacuum evaporation impregnation, ion exchange or the use of supercritical fluids [13, 14], have been proposed to render a homogeneous deposition of the catalytic active phase(s) without incurring in massive pore blockage. Impregnation-based methods are extensively used at the industrial level and are based on thermal treatments performed by calcination in air and reduction in $\mathrm{H}_{2}$. While a good number of reports have shown excellent control on the particle size distribution and internalization of metal and/or metal oxide nanoparticles after impregnation and gas treatment $[13,15]$, the number of works devoted to study the fate of the encased nanocatalysts after reaction is less extended, being especially remarkable the detailed studies carried out by Prieto et al. [16]. However, a facile methodology to produce nanosized particles with a good dispersion is still highly demanded. In the particular case of Pt/SBA-15 catalysts, a collection of alternative synthetic approaches more focused on the use of nanostructures and/or nanoemulsions has been lately explored in an attempt to balance easiness and control of hierarchy. Even though these methods cannot probably compete with industrial approaches, they still can be advantageous for other VOC removal applications such as indoor ambient purification. It is worth mentioning the feasible encapsulation of pre-formed Pt NPs during the hydrothermal formation of the SBA-15 mesostructures as synthetic protocol [17]. Nevertheless, this method requires an additional calcination step to remove the surfactant and the excess of stabilizer, and 
regrettably, uncontrolled Pt growth and/or coalescence cannot be prevented. Finally, the change of the overall surface charge of the ordered mesoporous silica materials using $\mathrm{pH}$ modifications [13] or after grafting additional functional groups have been also successfully tested $[8,18]$. In these latter cases, additional reducing agents, such as hydrogen [15], polyols [19] or formaldehyde [20] are required.

In this work, we present a facile procedure to produce Pt-SBA-15 catalysts using THPC as a novel reducing and stabilizing agent for the production of nanosized Pt NPs [21]. An amine-grafted SBA-15 support with sub-micron dimensions and straight porous channels has been developed [22]. The location and distribution of the metallic phase within the porous supports has been evaluated by conventional HR-TEM and via 3-D STEM-HAADF tomography. 3D HAADF-STEM tomography enables the nondestructive study of the interior of porous structures $[16,23]$. This technique is of paramount importance for heterogeneous catalysis, if a porous substrate were used because it enables the determination of the 3D shape, volume and connectivity of the pores, as well as the size and location of metallic particles inside the pores [24]. Concluding that 3-D HAADF-STEM tomography is very valuable since not only morphological but also chemical information can be extracted, eliminating the artefacts derived from diffraction contrast produced in 3D-TEM [23]. Likewise, their catalytic activity has been successfully tested on the total oxidation of n-hexane, selected as VOC model due to its low reactivity compared to other VOCs. The potential use of the tailored SBA-15 supports for industrial purposes is beyond the scope of the present study and a more plausible scenario of application based on the VOCs removal from indoor buildings has been taken into account [14]. 


\section{Experimental}

\subsection{Synthesis of rod-shaped SBA-15 mesoporous supports}

Rod-shaped SBA-15 supports were synthesized following a previously reported protocol [22]. Here, it is briefly described a typical synthesis: $1.2 \mathrm{~g}$ of Pluronic P123 and $0.014 \mathrm{~g}$ of $\mathrm{NH}_{4} \mathrm{~F}$ were dissolved in $40 \mathrm{~mL}$ of a freshly prepared $\mathrm{HCl}$ solution $(1.75$ M). The mixture was stirred at $20^{\circ} \mathrm{C}$ until the copolymer was totally dissolved. Subsequently, $9.5 \mathrm{~mL}$ of heptane were premixed with $2.75 \mathrm{~mL}$ of TEOS and the resulting mixture was then added to the micellar solution. The synthesis was kept under vigorous stirring for $4 \mathrm{~min}$ and then under static conditions for $10 \mathrm{~min}$. After reaction, the solution was transferred to a PTFE-lined autoclave for hydrothermal treatment at $100{ }^{\circ} \mathrm{C}$ for $24 \mathrm{~h}$. The resulting material was then filtered and washed with distilled water. Finally, the material was calcined at $550{ }^{\circ} \mathrm{C}$ for $5 \mathrm{~h}$ with a heating rate of $1{ }^{\circ} \mathrm{C}$ $\min ^{-1}$

\subsection{Amine-grafting of mesoporous supports and in-situ Pt loading}

The resulting mesoporous rods were post-grafted with amine terminal groups with the aid of (3-aminopropyl)triethoxysilane, following a previously described experimental protocol [25] (Fig. 1). Pt-loading was subsequently carried out by wet impregnation and in-situ formation of Pt NPs was produced by the reduction of chloroplatinic acid in the mesopores. Pt nanoparticles were formed at room temperature using the organophosphorous salt tetrakis-(hydroxymethyl)-phosphonium chloride (THPC) as simultaneous reducing and stabilizing agent [21]. In a typical synthesis, 100 $\mu \mathrm{L}$ of $\mathrm{H}_{2} \mathrm{PtCl}_{6}$ dispersed in $15 \mathrm{~mL}$ of double deionized (DDI) water containing $6.67 \mathrm{mg}$ of $\mathrm{NaOH}$ were added to $50 \mathrm{mg}$ of the amino-functionalized SBA-15 support. After stirring, $333 \mu \mathrm{L}$ of a $65 \mathrm{mM}$ THPC solution were added and left under stirring for 4 
days. The resulting product was thoroughly washed with DDI water, dried and stored for further use. The Pt nanoparticles generated were strongly attached to the mesoporous silica walls due to the electrostatic attraction between the electron-rich nitrogen present in the amino groups of the SBA-15 and the metal orbitals via their lone pairs [26]. The syntheses were performed using the facilities of the NANBIOSIS ICTS, more specifically by the Nanoparticle Synthesis Unit of the CIBER in BioEngineering, Biomaterials \& Nanomedicine (CIBER-BBN).

\section{Results and discussion}

\subsection{Platinum nanoparticles loaded in SBA-15 rods and their characterization}

SBA-15 rods were successfully synthesized and characterized by SEM, TEM XRD and $\mathrm{N}_{2}$ adsorption at $77 \mathrm{~K}$, thereby displaying an ordered channel distribution and nanosized dimensions; complete details are enclosed in Fig. S1 in the Supporting Information, SI. FT-IR spectroscopy further corroborated the presence of $v\left(\mathrm{CH}_{2}\right)$ stretching vibration modes in the functionalized supports, attributable to the alkyl part of APTES moieties condensed on the silica walls (see Fig. S2 in SI). Fig. 1 summarizes the three stages required to anchor the Pt nanoparticles to the SBA-15 pore walls. It must be highlighted that Pt nanoparticle precursors diffused through the mesopores and then crystallized inside the pores to provide a homogeneous distribution of $\mathrm{Pt}$ nanoparticles. Figure S3a-d shows representative (HR)-TEM and STEM-HAADF images of the SBA-15 nanorods after the Pt-loading step. Pt clusters with mean diameters below $1.4 \mathrm{~nm}$ (Fig. S3e) were obtained by the in-situ Pt loading procedure after reduction of the Pt salt in the presence of THPC. Pt NPs were anchored at the SBA-15 rods, following the mesopore channeling, which evidences their location inside the porous system rather than on its external surface (Fig. S3c). The presence of Pt is 
also supported by EDX analysis as shown in Fig. S3f. This organo-phosphorous THPC simultaneously acts as reducing agent and stabilizer as previously demonstrated for $\mathrm{Au}$ [27] and more recently for other noble-metal nanoparticles [21, 28]. Under basic conditions, THPC partially evolves to formaldehyde and hydrogen; two of the most suitable reducing agents for $\mathrm{Pt}[15,20]$. We hypothesize that after 4 days of reaction, the anchoring of $\mathrm{Pt}$ within the SBA-15 supports takes place via preliminary formation of R$\mathrm{NH}_{3}{ }^{+}-\left[\mathrm{PtCl}_{6}\right]^{2-}$ complexes which then undergo reduction [29]. A blank experiment with non-functionalized SBA-15 rods further corroborated the necessity of the amine terminal groups since most of the formed NPs remained unattached in the supernatant after centrifugation (see Fig. S4). The great control achieved in the metal loading, as well as the homogeneous particle size distribution of Pt can be argued in terms of the methodology of Pt growth by wet chemistry. The double role of THPC, and their derivatives, as reducing and stabilizing agents, enables an excellent control during the nucleation and growth stages of Pt NPs formation, achieving a particle size (1.4 nm) that is small enough not to hinder diffusion of reactants/products in a gas phase reaction through the pores $(8.7 \mathrm{~nm})$. Consequently, the metal loading methodology was clearly improved just by selecting a proper procedure based on wetchemistry instead of thermal treatments.

\subsection{Catalytic activity for n-hexane abatement}

The Pt/SBA-15 catalysts were tested in the total oxidation of $n$-hexane $(\geq 99.7 \%$

(GC) Sigma-Adrich), a linear alkane with reported lower reactivity than other VOC model compounds, such as aromatics or alcohols [30] (see also SI for experimental details). Fig. 2 shows the complete evolution of light-off curves after the pre-reduction treatment in $\mathrm{H}_{2}$ that further activates the catalyst and lowers $\mathrm{T}_{90}$ below $170{ }^{\circ} \mathrm{C}$. STEM- 
HAADF images of the particle size distribution after the reduction pre-treatment showed an increase in the number of less-ordered Pt clusters with sizes below $1.1 \mathrm{~nm}$ (see Fig. S3) in comparison with the as prepared sample. These clusters have been previous reported as extremely active in the oxidation of VOCs.

Although the presence of chloride and/or oxychlorinated species from the decomposition of Pt precursors has been previously addressed as a poisoning agent, influencing the catalytic activity towards the total oxidation of VOCs, our synthesis method differs from the more conventional impregnation approaches [2]. In principle, in our case, most of the excess of chlorine was expected to be washed away during the deposition/reduction of the Pt NPs carried out in aqueous solution. Hence, multiple XPS analyses of the initial catalysts, even at different etching rates, did not indicate any presence of Cl-related species (Fig. S5). Additionally, XPS analysis rendered similar XPS profiles indicative of a mixture of different valence states for Pt according to the binding energies of $\mathrm{Pt}_{4 / 2}$ and $\mathrm{Pt}_{7 / 2} \mathrm{f}_{5 / 2}$ doublets [31]. Fig. S6 illustrates a representative example of the combination of Pt in the metallic state (zero valent) detected by the doublet at 70.8 and $74.1 \mathrm{eV}$ and the presence of an additional $\mathrm{Pt}^{\delta+}$ oxidation state (where $0<\delta<2$ ) that can be either ascribed to partially oxidized $\mathrm{PtO}_{\mathrm{x}}$ species [31] or to small Pt clusters with a strong interaction with the support . This combination of oxidation states has been previously established for other active Pt-based catalysts, where the maximum activity was attributed to the less structured clusters. It was found that the pre-treatment in hydrogen also appears as the most determining step to activate the catalysts [20].

Regarding the catalyst's stability, the Pt/SBA-15 showed no evidence of deactivation in a long-term experiment carried out at $160{ }^{\circ} \mathrm{C}$ corresponding to a $40 \% \mathrm{n}$ hexane conversion after $70 \mathrm{~h}$ on reaction stream (Fig. S7). The high n-hexane 
conversion values achieved with the Pt/SBA-15 catalyst can be attributed to the excellent Pt dispersion on the larger pores of the rods, according to the work reported by Prieto et al. [23] for Co/SBA-15 catalysts. Finally, it is also worth mentioning that our catalytic results were similar or outperformed other Pt-based catalysts reported in the literature for the combustion of n-hexane working under similar space velocities as illustrated in Table 1.

Table 1.- Summary of selected works reporting the catalytic performance of the most active catalyst in the hexane oxidation reaction.

\begin{tabular}{|c|c|c|c|c|c|}
\hline Catalyst & $\operatorname{WHSV}\left(h^{-1}\right)$ & $\begin{array}{c}\text { [Hexane] } \\
\text { (ppm) }\end{array}$ & $\begin{array}{l}\mathrm{T}_{90} \\
\left({ }^{\circ} \mathrm{C}\right)\end{array}$ & $\begin{array}{c}\text { Max. } \\
\text { conversion, \% }\end{array}$ & Ref. \\
\hline $\mathrm{SS} / \mathrm{ZrO}_{2} / \mathrm{Pt}$ & 40 & 1500 & $\begin{array}{c}\text { Not } \\
\text { reached }\end{array}$ & 86 & [32] \\
\hline $\mathrm{SS} / \mathrm{ZrO}_{2} / \mathrm{La}_{2} \mathrm{O}_{3} / \mathrm{CuCo} / \mathrm{Pt}$ & 40 & 1500 & $\begin{array}{c}\text { Not } \\
\text { reached }\end{array}$ & 87 & [32] \\
\hline $\mathrm{Mn} / \mathrm{SiO}_{2}$ & 30000 & 360 & 270 & 98 & [9] \\
\hline $\mathrm{CoMn}-\mathrm{MS} / \mathrm{SiO}_{2}$ & 30000 & 360 & 265 & 100 & [9] \\
\hline Pt/ZSM5 & 315 & 200 & 275 & 97 & [14] \\
\hline $\mathrm{Pt} / \mathrm{ZY} 0.1$ & 315 & 200 & 225 & 100 & [14] \\
\hline $\mathrm{Pt} / \mathrm{ZY} 0.3$ & 315 & 200 & 223 & 100 & [14] \\
\hline $\mathrm{Au} / \mathrm{TiO}_{2}$ & 75 & 125 & 313 & 97 & {$[7]$} \\
\hline $\mathrm{Au} / \gamma-\mathrm{MnO}_{2}$ & 75 & 125 & 170 & 100 & [7] \\
\hline$\gamma-\mathrm{MnO}_{2}$ & 75 & 125 & 175 & 100 & [7] \\
\hline 5Mn15Co-MS & 14400 & 710 & 254 & 100 & [33] \\
\hline 5Co15Mn-MS & 14400 & 710 & 254 & 100 & [33] \\
\hline $0.4 \mathrm{Mn}-\mathrm{Pt} / \mathrm{MnO}_{\mathrm{x}}$ & 30 & 1500 & 279 & 95 & {$[6]$} \\
\hline $\begin{array}{l}\text { Asymetric membrane Pt/ } \\
\qquad \alpha-\mathrm{Al}_{2} \mathrm{O}_{3}\end{array}$ & 3300 & 2650 & 168 & 100 & [34] \\
\hline $\mathrm{Pt} / \mathrm{HRM}$ & 75.000 & 1000 & 240 & 100 & {$[35]$} \\
\hline Pt-SBA15 monolith & 80 & 200 & 186 & 100 & [8] \\
\hline Pt-SBA-15 NRs & 80 & 200 & 170 & 100 & This wor \\
\hline
\end{tabular}




\subsection{D HAADF-STEM tomography analysis}

A closer examination of the Pt-SBA-15 rods, after $70 \mathrm{~h}$ on reaction stream, shows the presence of larger nanoparticles in the size range of 6-8 nm (Fig. 3a) that can be attributed to the coalescence of nanoparticles during the activity test, mainly in the outer part of the mesoporous support, where the elimination of the amine functional group occurs more rapidly than inside the confined channels of the rods (Fig. S8). However, due to the 2D structural data obtained by STEM-HAADF images, it is challenging to elucidate if the sintered nanoparticles were located inside the mesopores or at the external particle surface. In spite of this, a large number of ultrasmall $\mathrm{Pt}$ nanoparticles $(1-2 \mathrm{~nm})$ were still observable. This fact explains the preservation of catalytic activity and suggests that the confinement of the nanoparticles within the mesochannels prevents the metal NPs from sintering (Fig. S8). Likewise, it has been reported that oxidative environments can also induce the splitting of Pt crystallites due to strain effects or the self-reduction due to thermal decomposition effects [20, 31].

3D HAADF-STEM tomography was conducted in order to elucidate the location of both Pt NPs $<1.4 \mathrm{~nm}$ and Pt NPs $>6 \mathrm{~nm}$ within the mesoporous channels. Fig. 3a presents a regular 2-D STEM-HAADF image of Pt-SBA-15, where the full thickness of the sample is integrated to yield this projection image and, thus, it is very difficult to get the information regarding the exact localization of the nanoparticles. In contrast, by analyzing the tomographic cross-sections taken at different orientations through the reconstructed volume shown in Fig. 3 b more information could be accessed. A simple comparison between Fig. 3a and $3 \mathrm{~b}$ reveals the large improvement in the signal-to-noise ratio, which enables attesting that the size distribution of the Pt NPs is bimodal. Thus, in addition to 5-7 nm Pt NPs (an approximate number of 45 NPs identified), a significant amount of 1-3 nm Pt nanoparticles situated on the internal walls of the porous structure 
of the SBA-15 rods was also identified. Unfortunately, it was not possible to provide an accurate number for the smaller NPs from further analyses of the reconstructed volume of the tomogram in Fig. 3, given their reduced size and the large amount of loaded NPs. By applying contrast gradients to the voxel matrix, the 3D model of the studied catalyst could be drawn for each component of the sample, i.e. for the porous structure and for the Pt nanoparticles. In the case of the SBA-15 rods, partial sintering was preferentially identified in the outer part of the mesoporous support (see section XY in Fig.3c and Fig. S9), where mesochannel confinement is ruled out. This can also be observed in Fig. 3d (slices taken through the $\mathrm{XZ}$ plane). The additional presence of multiple ultrasmall $\mathrm{Pt}$ nanoparticles below $2 \mathrm{~nm}$ further corroborates the stability of these catalysts even after a significant number of hours on reaction stream and the minimal effect of the mild reaction conditions. Consequently, these analyses confirm that mesochannels are beneficial as a barrier against catalyst sintering and a high grade of Pt loading was located at the pore system rather than on the external particle surfaces.

\section{Conclusions}

Nanosized Pt-decorated SBA-15 rods with excellent catalytic performance towards the total oxidation of n-hexane have been successfully synthesized following a facile synthesis method that involves the use of THPC. This phosphorous compound can simultaneously induce the in-situ growth of Pt NPs by the reduction of Pt precursors and promote their colloidal stabilization. A better catalytic performance has been obtained for the rod-shaped supports in comparison with previously reported mesoporous supports. This fact has been attributed to a good dispersion of the nanoparticles into the large pore channels, elevated surface areas and enhanced accessibility to active sites. Finally, a 3D STEM model of the Pt/SBA-15 nanorods 
catalysts has been presented with the aid of tomographic tools that corroborate the presence and confinement of the Pt nanoparticles within the mesoporous channels of the catalytic support and their limited coalescence even after multiple hours on reaction stream.

\section{Acknowledgements}

Financial support from the MICINN through the MAT2011-24988 grant, the European Research Council (ERC-Advanced Grant-HECTOR), People Program (CIG-Marie Curie Actions, REA grant agreement no. 321642 and no. 294094) and the CIBER-BBN. These TEM measurements were supported by the European Union Seventh Framework Program under Grant Agreement 312483 - ESTEEM2 (Integrated Infrastructure Initiative - I3).

\section{References}

[1] J.J. Spivey, Ind. Eng. Chem. Res. 26 (1987) 2165-2180.

[2] A. Borgna, T.F. Garetto, C.R. Apesteguia, F. Le Normand, B. Moraweck, J Catal $186(1999) 433-441$.

[3] S. Ordonez, L. Bello, H. Sastre, R. Rosal, F.V. Diez, Appl. Catal. B-Environ. 38 (2002) 139-149.

[4] R. Pereniguez, J.L. Hueso, F. Gaillard, J.P. Holgado, A. Caballero, Catal. Lett. $142(2012) 408-416$.

[5] C. Cellier, V. Ruaux, C. Lahousse, P. Grange, E.M. Gaigneaux, Catal Today 117 (2006) 350-355.

[6] M. Anic, N. Radic, B. Grbic, V. Dondur, L. Damjanovic, D. Stoychev, P. Stefanov, Appl. Catal. B-Environ. 107 (2011) 327-332. 
[7] C. Cellier, S. Lambert, E.M. Gaigneaux, C. Poleunis, V. Ruaux, P. Eloy, C. Lahousse, P. Bertrand, J.P. Pirard, P. Grange, Appl. Catal. B-Environ. 70 (2007) 406416.

[8] L. Uson, M.G. Colmenares, J.L. Hueso, V. Sebastian, F. Balas, M. Arruebo, J. Santamaria, Catal. Tod. 227 (2014) 179-186.

[9] S. Todorova, A. Naydenov, H. Kolev, J.P. Holgado, G. Ivanov, G. Kadinov, A. Caballero, Appl Catal a-Gen 413 (2012) 43-51.

[10] L.C. Liu, U. Diaz, R. Arenal, G. Agostini, P. Concepcion, A. Corma, Nat Mater $16(2017) 132-138$.

[11] C.J. Jia, F. Schuth, Phys Chem Chem Phys 13 (2011) 2457-2487.

[12] A. Vinu, V. Murugesan, W. Bohlmann, M. Hartmann, J. Phys. Chem. B 108 (2004) 11496-11505.

[13] L. Jiao, J.R. Regalbuto, J Catal 260 (2008) 342-350.

[14] N. Navascues, M. Escuin, Y. Rodas, S. Irusta, R. Mallada, J. Santamaria, Ind. Eng. Chem. Res. 49 (2010) 6941-6947.

[15] M.J. Jacinto, R. Landers, L.M. Rossi, Catal Commun 10 (2009) 1971-1974.

[16] G. Prieto, J. Zecevic, H. Friedrich, K.P. de Jong, P.E. de Jongh, Nat Mater 12 (2013) 34-39.

[17] R.M. Rioux, H. Song, J.D. Hoefelmeyer, P. Yang, G.A. Somorjai, J. Phys. Chem. B 109 (2005) 2192-2202.

[18] M. Colilla, I. Izquierdo-Barba, S. Sanchez-Salcedo, J.L.G. Fierro, J.L. Hueso, M. Vallet-Regi, Chem. Mat. 22 (2010) 6459-6466.

[19] J.J. Zhu, X.A. Xie, S.A.C. Carabineiro, P.B. Tavares, J.L. Figueiredo, R. Schomacker, A. Thomas, Energy Environ. Sci. 4 (2011) 2020-2024. 
[20] Y. Tang, S.J. Miao, H.N. Pham, A. Datye, X.M. Zheng, B.H. Shanks, Appl Catal a-Gen 406 (2011) 81-88.

[21] J.L. Hueso, V. Sebastian, A. Mayoral, L. Uson, M. Arruebo, J. Santamaria, RSC Adv. 3 (2013) 10427-10433.

[22] E.M. Johansson, M.A. Ballem, J.M. Cordoba, M. Oden, Langmuir 27 (2011) 4994-4999.

[23] S. Moldovan, R. Arenal, O. Ersen, in: F.L. Deepak, A. Mayoral, R. Arenal (Eds.), Advanced Transmission Electron Microscopy: Applications to Nanomaterials, Springer International Publishing, Cham, 2015, pp. 171-205.

[24] U. Ziese, K.P. de Jong, A.J. Koster, Appl Catal a-Gen 260 (2004) 71-74.

[25] F. Balas, M. Manzano, M. Colilla, M. Vallet-Regí, Acta Biomater. 4 (2008) 514522.

[26] A.H. Pakiari, Z. Jamshidi, J. Phys. Chem. A 111 (2007) 4391-4396.

[27] D.G. Duff, A. Baiker, P.P. Edwards, Langmuir 9 (1993) 2301-2309.

[28] L. Uson, V. Sebastian, A. Mayoral, J.L. Hueso, A. Eguizabal, M. Arruebo, J. Santamaria, Nanoscale 7 (2015) 10152-10161.

[29] R. Dobrowolski, M. Oszust-Cieniuch, J. Dobrzynska, M. Barczak, Colloid Surf. A-Physicochem. Eng. Asp. 435 (2013) 63-70.

[30] A. O’Malley, B.K. Hodnett, Catal. Tod. 54 (1999) 31-38.

[31] S. Porsgaard, L.R. Merte, L.K. Ono, F. Behafarid, J. Matos, S. Helveg, M. Salmeron, B.R. Cuenya, F. Besenbacher, ACS Nano 6 (2012) 10743-10749.

[32] T. Novakovic, N. Radic, B. Grbic, T. Marinova, P. Stefanov, D. Stoychev, Catal Commun 9 (2008) 1111-1118.

[33] S. Todorova, H. Kolev, J.P. Holgado, G. Kadinov, C. Bonev, R. Pereniguez, A. Caballero, Appl. Catal. B-Environ. 94 (2010) 46-54. 
[34] S. Zalamea, M.P. Pina, A. Villellas, M. Menendez, J. Santamaria, React Kinet Catal L 67 (1999) 13-19.

[35] S.C. Kim, S.W. Nahm, Y.-K. Park, Journal of Hazardous Materials 300 (2015) 104-113. 


\section{Figure Captions}

Figure 1. Scheme of the different steps followed during the synthesis of Pt/SBA-15 Rods.

Figure 2. Catalytic activity of the Pt/SBA-15 Rods (3 wt\% Pt loading) in terms of $n$ hexane conversion versus temperature after a reduction pre-treatment in $\mathrm{H}_{2}$.

Figure 3. (a) Low magnification (LM)-HAADF image from the tilt series recorded on the Pt/SBA-15 Nanorods after $70 \mathrm{~h}$ on reaction stream; (b) 3D model of the nanoparticle obtained by a segmentation procedure from a reconstructed HAADF-STEM tilt series, with the mean surface of the mesoporous SAB-15 material in blue and the $\mathrm{Pt}$ nanoparticles in red respectively; (c) Typical longitudinal slice taken through the XY plane illustrating the presence of the Pt nanoparticles within the mesoporous channels (different coloured arrows illustrate different Pt sizes); (d) Slice 1 taken through the XZ plane showing more clearly the presence of the small Pt NPs $1.5-3 \mathrm{~nm}$. The different planes (marked as 1-4) are highlighted in Fig. 3 (b). Slices 2-4 are plotted separately in the SI. 


\section{SBA-15 Synthesis Functionalization Pt NPs growth}

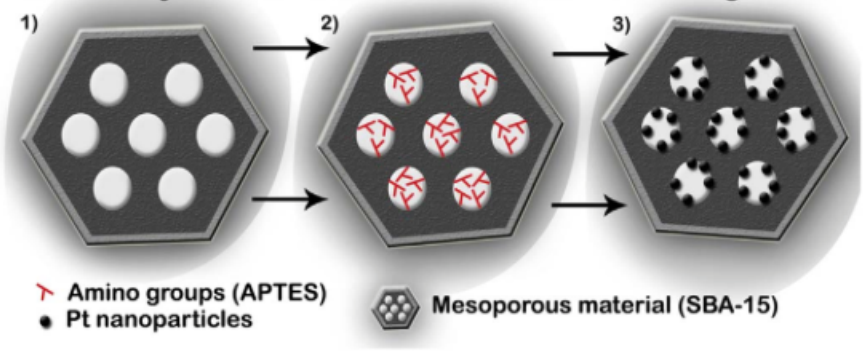

Fig. 1. Scheme of the different steps followed during the synthesis of Pt/SBA-15 Rods.
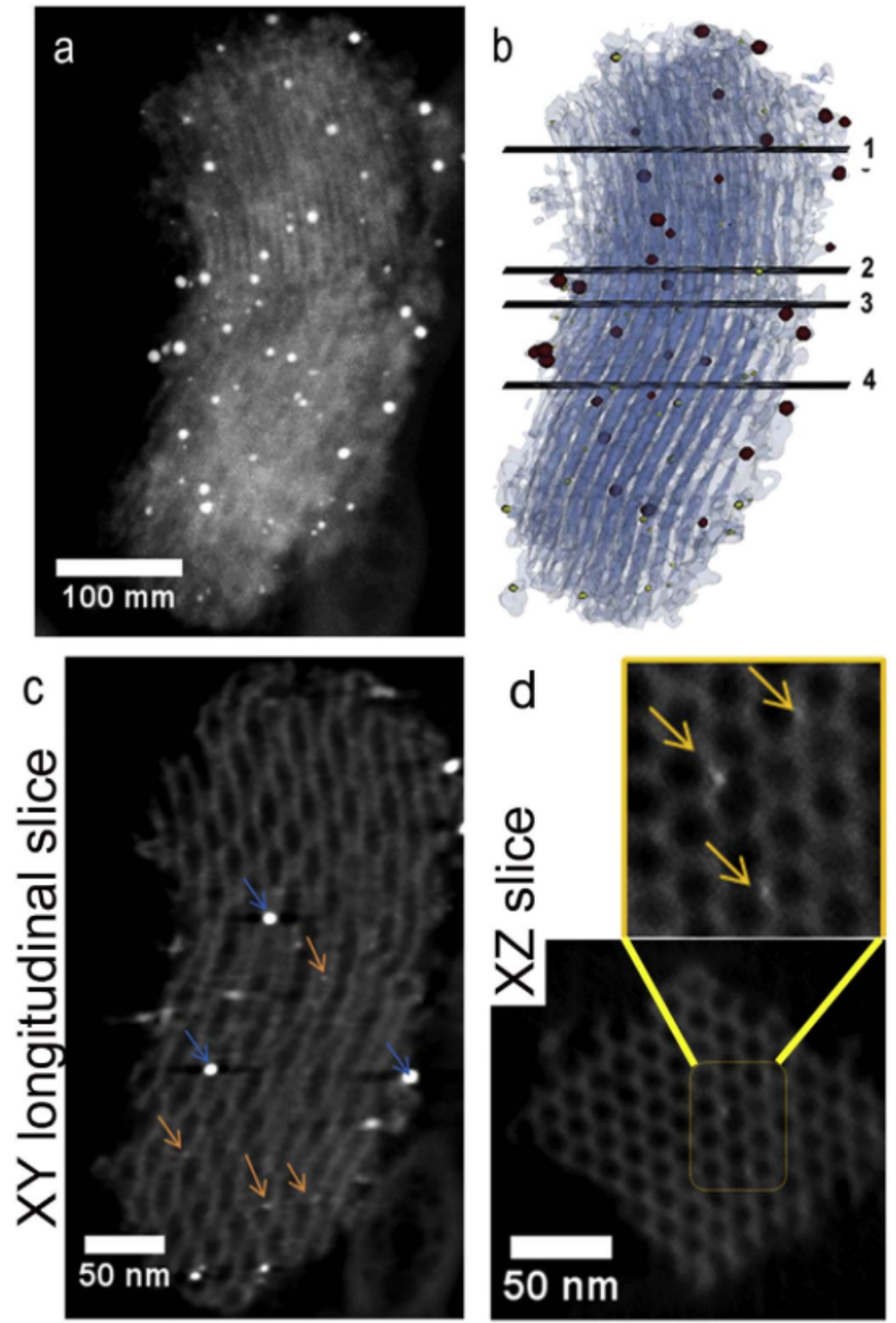

Fig. 3. (a) Low magnification (LM)-HAADF image from the tilt series recorded on the Pt/ SBA-15 Nanorods after $70 \mathrm{~h}$ on reaction stream; (b) 3D model of the nanoparticle obtained by a segmentation procedure from a reconstructed HAADF-STEM tilt series, with the mean surface of the mesoporous SAB-15 material in blue and the Pt nanoparticles in red respectively; (c) Typical longitudinal slice taken through the XY plane illustrating the presence of the Pt nanoparticles within the mesoporous channels (different coloured arrows illustrate different Pt sizes); (d) Slice 1 taken through the $\mathrm{XZ}$ plane showing more clearly the presence of the small Pt NPs $1.5-3 \mathrm{~nm}$. The different planes (marked as 1-4) are highlighted in (b). Slices $2-4$ are plotted separately in the SI. (For interpretation of the references to colour in this figure legend, the reader is referred to the web version of this article.)
Fig. 2. Catalytic activity of the Pt/SBA-15 Rods ( 3 wt $\%$ Pt loading) in terms of $n$-hexane conversion versus temperature after a reduction pre-treatment in $\mathrm{H}_{2}$. 